\title{
Robust shallow water models
}

\author{
Reinhard Hinkelmann $^{1} \cdot$ Qiuhua Liang $^{2} \cdot$ Vadym Aizinger $^{3} \cdot$ Clint Dawson $^{4}$
}

Received: 27 September 2015/ Accepted: 2 October 2015/Published online: 11 October 2015

(C) Springer-Verlag Berlin Heidelberg 2015

Shallow water models have been used extensively for decades with various applications in hydroscience and engineering such as flood prediction and management, river restoration and engineering, environmental hydraulics and morphodynamics. In recent years, there has been a special focus on developing robust solvers which are capable of simulating a wide range of complex hydrodynamic problems including dam breaks (shock propagation, wetting and drying, small water depths), as well as flow transitions (subcritical-supercritical flow and vice versa, hydraulic jumps). In addition to classical finite-volume and finite-element schemes, newer methods such as smoothed particle hydrodynamics (SPH) are now being applied and extended. Furthermore, we have seen rapid development in surveying techniques (e.g. airborne and terrestrial laser scanning) which describe the geometry and physical properties of our systems, providing us with increasingly high-resolution information. Substantial improvements have also been made in high-performance computing, with notable advances including Graphic Processing Unit (GPU)-based parallel computing as well as scaling methods, relying, for example, on friction- or porosity-based approaches. Linkage of robust numerical methods, high-

Reinhard Hinkelmann

reinhard.hinkelmann@wahyd.tu-berlin.de

1 Chair of Water Resources Management and Modeling of Hydrosystems, TU Berlin, Berlin, Germany

2 Chair of Hydrosystems Modelling, Newcastle University, Newcastle upon Tyne, UK

3 Chair of Applied Mathematics 1, University of ErlangenNuernberg, Erlangen, Germany

4 Computational Hydraulics Group, The University of Texas, Austin, TX, USA resolution data, high-performance computing, scaling methods and new information and communication technologies will lead to the next generation of shallow water models opening up new application fields such as rainfallrunoff simulation in urban and rural catchments, real-time flood and tsunami prediction and management, as well as the spreading of impulse water waves generated by landslides.

In view of these developments, we have encouraged submissions on both the development of numerical models and interesting applications, for the $X X$ International Conference on Computational Methods in Water Resources held on June 10-13, 2014 in Stuttgart, Germany, especially for the sessions on 'Robust shallow water models', 'Numerical methods for waves, circulation and transport in the coastal Ocean' and 'High-performance computing, visualization and scientific workflow'. This special issue contains papers which have been selected by the guest editors among about 20 contributions. The corresponding authors have been offered the opportunity to contribute to this special issue through a paper about their conference contribution (abstract, oral, or poster presentation). The papers have all undergone a full peer-review process as regular journal submissions and, finally, we are happy to present this special issue consisting of ten papers. This special issue widens the already existing journal focus on shallow water models and their applications, see, for example, Delfs et al. (2013), Shi et al. (2014), Carbajal et al. (2014) or Huang et al. (2014).

This special issue starts with two contributions dealing with floods. Beisiegel and Behrens (2015) have developed a robust discontinuous Galerkin (DG) model using special basis functions and a slope limiter. Flooding and drying is simulated through applying a stable scheme, and the model's advantages are demonstrated in a numerical study. 
An SPH approach for urban flood modelling presented by Liang et al. (2015a) had increased its computational efficiency using modern GPUs. The model's capabilities and enhanced computational efficiency are demonstrated by applying it to a field-scale hypothetical urban flood event.

Two further papers are dealing with rainfall-runoff simulations. Lacasta et al. (2015) introduce an efficient GPU implementation of the 2D shallow water equations on unstructured grids. They analyse the influence of the mesh resolution on the computational performance and the model accuracy concluding that their tool is very beneficial for environmental simulation of hydrological processes. Idealised catchments with and without obstacles such as buildings are analysed and verified by comparing 2D numerical with analytical and experimental results in Liang et al. (2015b). They determine dimensional S-curves which generically depict the relation between rainfall and runoff.

A further focus of this special issue is represented by three contributions which consider transport processes in addition to shallow water flow. Morales-Hernandez et al. (2015) present a Riemann-based coupled 1D-2D shallow water flow and solute transport model. Inundation can be simulated correctly and the results of the coupled 1D-2D model are compared with those of the complete 2D model also considering CPU time. A residual distribution and a finite-volume scheme have been developed in the framework of the Telemac2D system by Pavan et al. (2015). The two schemes are compared in several test cases simulating pollutant transport on unstructured grids considering numerical diffusion, mass conservation, and monotonicity. Ortiz et al. (2015) have developed a continuous finite element model for shallow water flow and bedload sediment transport permitting computations without spurious mass exchanges and oscillations even under severe conditions. They apply their model to dam-break problems with erodible beds and to flood-induced morphodynamics in a real river.

Finally, there are three contributions from other fields of robust shallow water models. Özgen et al. (2015) present an upscaling approach for the shallow water equations based on a novel roughness formulation which accounts for microtopography. They point out the advantages as well as drawbacks of their scheme in different simple and real-case applications considering solution accuracy and speedup in simulation time. An algorithm to model the spreading of impulse water waves generated by submarine landslides using adaptive grids has been developed by Shokina and Aizinger (2015). Numerical and experimental results are compared for different cases. Hou et al. (2015) discuss absorbing boundary conditions to reduce spurious waves which are caused by missing information from outside of the domain. They suggest a domain extension and give recommendations on how to do it, demonstrating the model results for two field-scale tsunami events.

The guest editors hope you have fun reading the papers and gratefully acknowledge the contributions of the authors and the reviewers and the support of Olaf Kolditz, the Editor-in-Chief for Special Issues.

\section{References}

Beisiegel N, Behrens J (2015) Quasi-nodal third-order Bernstein polynomials in a discontinuous Galerkin model for flooding and drying. Environ Earth Sci. doi:10.1007/s12665-015-4745-4

Carbajal N, Dworak JA, Montano-Ley Y, Noyola-Medrano C (2014) Influence of friction on erosion and accretion processes in the Yavaros Bay, Gulf of California. Environ Earth Sci 72(2):429-440

Delfs JO, Wang WQ, Kalbacher T, Singh AK, Kolditz O (2013) A coupled surface/subsurface flow model accounting for air entrapment and air pressure counterflow. Environ Earth Sci 69(2):395-414

Hou J, Liang Q, Xia X (2015) Robust absorbing boundary conditions for shallow water flow models. Environ Earth Sci. doi:10.1007/ s12665-015-4743-6

Huang JC, Gao JF, Hormann G, Fohrer N (2014) Modeling the effects of environmental variables on short-term spatial changes in phytoplankton biomass in a large shallow lake, Lake Taihu. Environ Earth Sci 72(9):3609-3621

Lacasta A, Morales-Hernandez M, Murillo J, Garcia-Navarro P (2015) GPU implementation of the 2D shallow water equations for the simulation of rainfall/runoff events. Environ Earth Sci. doi:10.1007/s12665-015-4215-Z

Liang D, Özgen I, Hinkelmann R, Xiao Y, Chen JM (2015a) Shallow water simulation of overland flows in idealised catchments. Environ Earth Sci. doi:10.1007/s12665-015-4744-5

Liang Q, Xia X, Hou J (2015b) Efficient urban flood simulation using a GPU-accelerated SPH model. Environ Earth Sci. doi:10.1007/ s12665-015-4753-4

Morales-Hernandez M, Lacasta A, Murillo J, Brufau P, GarcíaNavarro P (2015) A Riemann coupled edge (RCE) 1D-2D finite volume inundation and solute transport model. Environ Earth Sci. doi:10.1007/s12665-015-4754-3

Ortiz P, Anguita J, Riveiro M (2015) Free surface flows over partially erodible beds by a continuous finite element method. Environ Earth Sci. doi:10.1007/s12665-015-4730-y

Özgen I, Teuber K, Simons F, Liang D, Hinkelmann R (2015) Upscaling the shallow water model with a novel roughness formulation. Environ Earth Sci. doi:10.1007/s12665-015-4726-7

Pavan S, Ata R, Hervouet JM (2015) Finite volume schemes and residual distribution schemes for pollutant transport on unstructured grids. Environ Earth Sci. doi:10.1007/s12665-015-4760-5

Shi J, Tong CF, Yan YX, Luo XQ (2014) Influence of varying shape and depth on the generation of tidal bores. Environ Earth Sci 72(7):2489-2496

Shokina N, Aizinger V (2015) On numerical modelling of impulse water waves generated by submarine landslides. Environ Earth Sci. doi:10.1007/s12665-015-4746-3 